\title{
Volume of Interest
}

National Cancer Institute

\section{Source}

National Cancer Institute. Volume of Interest. NCI Thesaurus. Code C95001.

A user-selected volume within a 3D image. The choice is guided by knowledge that activities concentrations for the voxels within the boundary contour of this region will be used in quantifying it. 